\title{
Relay Positioning for Multicast Relay Networks
}

\author{
Mohit Thakur and Gerhard Kramer \\ Institute for Communications Engineering \\ Technische Universität München, Germany \\ mohit.thakur@tum.de,gerhard.kramer@tum.de
}

\begin{abstract}
The relay positioning problem is addressed for low signal-to-noise ratio Gaussian multicast relay networks where the objective is to maximize the multicast flow. A network coding based decode-and-forward (DF) strategy is presented that achieves strictly larger rates than a previously-studied routing based DF strategy. Properties of the multicast flow function are analyzed and efficient solutions are presented.
\end{abstract}

\section{INTRODUCTION}

Consider the problem of relay positioning for a low signalto-noise ratio (SNR) Gaussian multicast relay channel (MRC) consisting of a single source, a single relay with half-duplex constraints and multiple destinations. The goal is to maximize the multicast flow. Relay positioning has been addressed in various scenarios, e.g. to maximize the coverage in an area for a given rate [1]. A detailed examination of achievable rates for the Gaussian relay channel using different cooperative strategies was done in [2], and their performance was compared for some relay positions.

In [3], Thakur et al. considered the relay positioning problem in the context of maximizing the multicast flow for a routing based decode-and-forward (DF) strategy. The problem was formulated as a flow optimization problem over hypergraphs and was shown to be non-convex in general. In this paper, we present a network coding based DF strategy that achieves strictly larger rates than the routing based DF strategy in [3]. The network coding based DF strategy achieves the same rate as the non-coherent DF lower bound derived in [4] for the Gaussian relay channel. We show that the joint multicast flow maximization and relay positioning problem can be formulated as a quasi-concave optimization program with a single optimizing variable (the position of the relay) that can be solved efficiently. Throughout the paper, we assume noncoherent communication and that the relay is half-duplex.

The paper is organized as follows. Section II describes the Gaussian MRC. Sections III and IV present the routing DF strategy from [3] and introduce the network coding DF strategy, respectively. Section V compares the rates achieved by the two strategies. Section VI concludes the paper.

\section{Gaussian Mutlicast Relay Channel}

Consider a network with a source $s$, a relay $r$, and a set of destinations $T=\left\{t_{1}, . ., t_{m}\right\}$ with the node set denoted by $\mathcal{N}=\{s, r\} \cup T$. The nodes are positioned on the twodimensional Euclidean plane and $\mathbf{x}_{u}$ denotes the position of node $u \in \mathcal{N}$.

For sets $\mathcal{X}, \mathcal{X}_{r}, \mathcal{Y}_{r}, \mathcal{Y}_{1}, \mathcal{Y}_{2}, . ., \mathcal{Y}_{m}$, a MRC has the source input $x \in \mathcal{X}$, relay output $y_{r} \in \mathcal{Y}_{r}$, relay input $x_{r} \in \mathcal{X}_{r}$ and

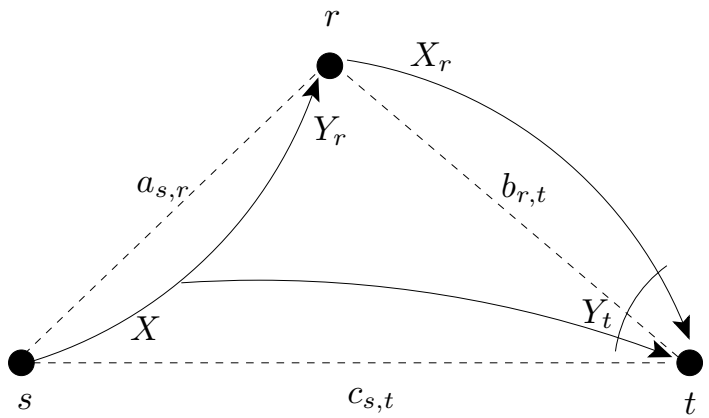

Fig. 1: Single destination relay channel.

destination outputs $y=\left(y_{1}, . ., y_{m}\right) \in \mathcal{Y}$, where $\mathcal{Y}=\mathcal{Y}_{1} \times$ ..$\times \mathcal{Y}_{m}$. The channel is governed by a conditional probability distribution $p\left(y, y_{r} \mid x_{r}, x\right)$.

Consider a source codebook with codewords of length $n$ that are indexed by messages in the set $\mathcal{M}=\left\{1,2, . ., 2^{n R}\right\}$, where $R$ is the rate or the multicast flow. The message $M$ is drawn uniformly from $\mathcal{M}$ and the source transmits the codeword indexed by $M$. The relay maps its past channel outputs to its channel inputs via

$$
x_{r, i}=\mathfrak{r}_{i}\left(y_{r, 1}, \ldots, y_{r, i-1}\right), \quad 1 \leq i \leq n
$$

where $\mathfrak{r}_{i}$ is some function. Decoding functions $\mathfrak{t}_{j}: \mathcal{Y}_{j} \longrightarrow$ $\mathcal{M}_{j}$ map the received sequences $y_{j}$ to message estimates $\hat{M}_{j} \in \mathcal{M}_{j}, \forall t_{j} \in T$. The rate $R$ is said to be achievable if, for sufficiently large $n$, there exist codebooks, relay encoding functions, and decoding functions such that $\operatorname{Pr}\left[\bigcup_{j=1}^{m}\left\{\hat{M}_{j} \neq M\right\}\right] \leq \delta$ for any $\delta>0$. The goal is to find the supremum of the achievable $R$.

We focus attention on additive white Gaussian noise (AWGN) MRCs with a simple path-loss model. All channel alphabets are the complex numbers and the outputs at time $i \geq 1$ (see Fig. 1) are

$$
\begin{aligned}
& Y_{r, i}=a_{s, r} X_{i}+Z_{r, i} \\
& Y_{j, i}=c_{s, j} X_{i}+b_{r, j} X_{r, i}+Z_{j, i}, \quad 1 \leq j \leq m
\end{aligned}
$$

where $a_{s, r}, b_{r, j}$ and $c_{s, j}$ are channel gains. The relay and destination noise processes $\left\{Z_{r, i}\right\}$ and $\left\{Z_{j, i}\right\}$ have i.i.d. proper complex Gaussian noise variables with variance $N$. The channel gains satisfy the relations

$$
a_{s, r}=\frac{h_{s, r}}{D_{s, r}^{\alpha / 2}}, \quad b_{r, j}=\frac{h_{r, j}}{D_{r, j}^{\alpha / 2}}, \quad c_{s, j}=\frac{h_{s, j}}{D_{s, j}^{\alpha / 2}},
$$

for $1 \leq j \leq m$, where the $h_{u, v}$ are (real) fading gains and $D_{u, v}=\left\|\mathbf{x}_{u}-\mathbf{x}_{v}\right\|$ is the Euclidean distance between nodes 


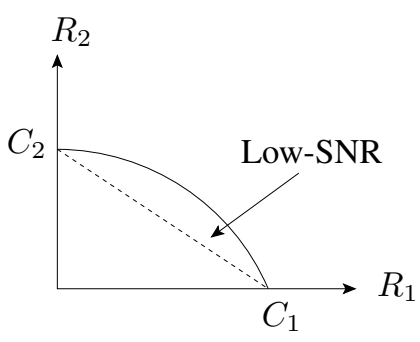

(a)

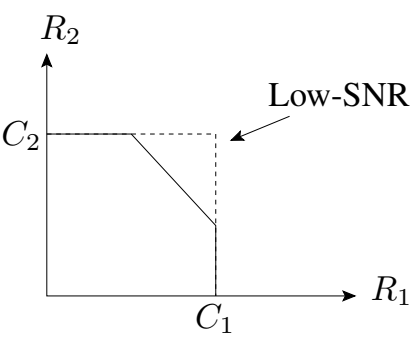

(b)
Fig. 2: Figures (a) and (b) show the rate regions of the broadcast and MAC channel, respectively, for two user case.

$u$ and $v$. The number $\alpha$ satisfies $\alpha \geq 2$ and is the path loss exponent. The average error probability for decoding at destination $t_{j} \in T$ is

$$
\mathrm{P}_{e, j}^{n}=\frac{1}{2^{n R}} \sum_{k=1}^{2^{n R}} \operatorname{Pr}\left[\hat{M}_{j} \neq M \mid M=k\right] .
$$

There are average block power constraints

$$
\frac{1}{n} \sum_{i=1}^{n} x_{i}^{2} \leq P_{s}, \quad \frac{1}{n} \sum_{i=1}^{n} x_{r, i}^{2} \leq P_{r}
$$

where $P_{r}$ and $P_{s}$ are non-negative real numbers.

\section{ROUTING DF STRATEGY}

The routing based DF strategy in [3] uses superposition coding for broadcasting. We review results on AWGN broadcast channels (BCs) and multiaccess channels (MACs).

\section{A. Broadcast}

Consider an AWGN BC where a source $s$ with power $P_{s}$ transmits $W$ symbols per second in a bandwidth of $W$ Hertz. The source has a message of rate $R_{j}$ for destination $t_{j}, 1 \leq$ $j \leq m$. Suppose destination $t_{j}$ is better off than destination $t_{k}$, i.e. $c_{s, j} \geq c_{s, k}$ for $1 \leq j<k \leq m$. It is well known that superposition coding achieves capacity for scalar BCs (see [5] and [6]), and node $t_{j}$ may subtract off the estimated signals of all $t_{k}, k>j$, from its received signal before decoding. The resulting rates in nats per second are

$$
W R_{j}=W \log \left(1+\frac{\lambda_{j} c_{s, j}^{2} P_{s}}{W N+\sum_{k=1}^{j-1} c_{s, k}^{2} \lambda_{k} P_{s}}\right)
$$

for $1 \leq j \leq m$, where $\sum_{j=1}^{m} \lambda_{j}=1$ and $\lambda_{j} \geq 0$. In the high bandwidth / low-SNR regime, the rates become (see Fig. 2a)

$$
\lim _{W \longrightarrow \infty} W R_{j} \lesssim \frac{\lambda_{j} c_{s, j}^{2} P_{s}}{N} .
$$

The rate region becomes a time-sharing region at low-SNR.

\section{B. Multiaccess}

Consider two sources $s_{1}$ and $s_{2}$ transmitting to common destination $t$ with powers $P_{s, 1}$ and $P_{s, 2}$, respectively. The

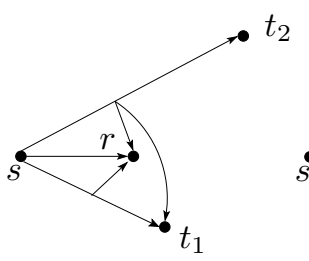

(a)

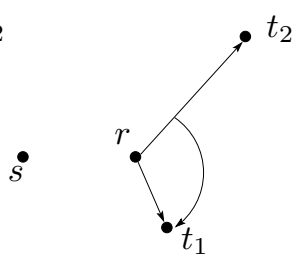

(b)

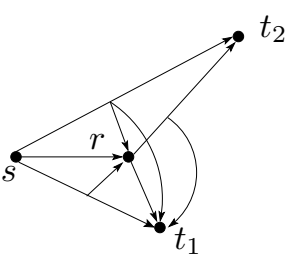

(c)
Fig. 3: $a_{s, r}>a_{s, 1}>a_{s, 2}$ and $b_{r, 1}>b_{r, 2}$. (a): Broadcast channel from $s$ decomposes into hyperarcs $(s, r),\left(s, r, t_{1}\right)$ and $\left(s, r, t_{1}, t_{2}\right)$. (b): Similarly, for $r$ we have $\left(r, t_{1}\right)$ and $\left(r, t_{1}, t_{2}\right)$. (c): Low-SNR Hypergraph: concatenation of (a) and (b).

available bandwidth is again $W$ Hertz. Let $R_{j, t}$ be the achievable rates between $s_{j}$ and $t$, so that we have standard bounds

$$
\begin{aligned}
& W R_{j, t} \leq W \log \left(1+\frac{c_{j, t}^{2} P_{s, j}}{W N}\right), \quad j \in\{1,2\} \\
& W\left(R_{1, t}+R_{2, t}\right) \leq \sum_{j \in\{1,2\}} W \log \left(1+\frac{c_{j, t}^{2} P_{s, j}}{W N}\right) .
\end{aligned}
$$

In the high bandwidth / low-SNR regime, the rates become

$$
\begin{aligned}
& W R_{1, t} \lesssim \frac{c_{1, t}^{2} P_{s, 1}}{N}, W R_{2, t} \lesssim \frac{c_{2, t}^{2} P_{s, 2}}{N} \\
& W\left(R_{1, t}+R_{2, t}\right) \lesssim \frac{c_{1, t}^{2} P_{s, 1}}{N}+\frac{c_{2, t}^{2} P_{s, 2}}{N} .
\end{aligned}
$$

The rate region becomes a rectangle at low-SNR (see Fig. 2b).

\section{Low-SNR Hypergraph Model}

Consider the hypergraph approach in [3], which is obtained by concatenating the broadcast hypergraphs. For the node set $\mathcal{N}$ we have two broadcast channels corresponding to nodes $s$ and $r$, and $m$ MAC channels corresponding to destinations $t_{j} \in T$. Figure 3 shows an example with $\mathcal{N}=\left\{s, r, t_{1}, t_{2}\right\}$.

Consider a path from $s$ to all the destinations, for instance $\left\{(s, r),\left(r, t_{1}, t_{2}\right)\right\}$ in Fig. 3c. Recall that the relay has a half-duplex constraint. Consider the following transmission/reception schedules.

(i) Schedule with no interference: In the first time slot the source transmits over the hyperarc $(s, r)$ and the relay decodes. The relay transmits in the second time slot over the hyperarc $\left(r, t_{1}, t_{2}\right)$ as shown in Fig. 4 . Note that in the first slot, only the relay listens as only the hyperarc $(s, r)$ is in use. Although we focus on the low-SNR regime, the above schedule is valid for other regimes as well.

\begin{tabular}{c||cc} 
Time $\backslash$ Node & $s$ & $r$ \\
\hline$i$ & 1 & 0 \\
$i+1$ & 0 & 1
\end{tabular}

(a) Transmission.

\begin{tabular}{c||ccc} 
Time $\backslash$ Node & $r$ & $t_{1}$ & $t_{2}$ \\
\hline$i$ & 1 & 0 & 0 \\
$i+1$ & 0 & 1 & 1
\end{tabular}

(b) Reception.
Fig. 4: Schedule with no interference. A "0" means that the device is inactive, while a " 1 " means that the device is active.

(ii) Schedule with interference: Suppose the source transmits in the second time slot as shown in Fig. 5. Thus, in the second time slot the destinations receive signals 
from both the transmitters and their achievable rates are constrained by the MAC bounds (8)-(9). However, in the low-SNR regime the effect of interference is negligible.

\begin{tabular}{|c|c|c|c|c|c|c|}
\hline Time $\backslash$ Node & $s$ & $r$ & Time $\backslash$ Node & $r$ & $t_{1}$ & $t_{2}$ \\
\hline$i$ & 1 & 0 & 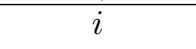 & 1 & 0 & 0 \\
\hline$i+1$ & 1 & 1 & $i+1$ & 0 & 1 & 1 \\
\hline
\end{tabular}

(a) Transmission.

(b) Reception.

Fig. 5: Schedule with interference.

\section{Relay Positioning}

We address the question: What relay position maximizes the multicast flow? As in [3], for the low-SNR regime the problem is formulated as an optimization program. The program is nonconvex in general, as was shown in [3], and the optimization can be computationally expensive as different relay positions require using different hypergraphs. We compare the performance of this strategy against the network coding based DF strategy in section $\mathrm{V}$.

\section{Network Coding DF StRategy}

We use a network coding based transmission strategy for which all destinations always listen. As a result, we do not get a hypergraph but a link-based graph structure (see Fig. 6).

Consider the AWGN MRC described in Section II. Using DF block-Markov coding, we get the following achievable rates

$$
R_{j}=\max _{p\left(x_{s}, x_{r}\right)}\left\{\min \left(I\left(X_{s} ; Y_{r} \mid X_{r}\right), I\left(X_{s} X_{r} ; Y_{j}\right)\right)\right\}
$$

for $1 \leq j \leq m$. We can thus write the rates $\left(W R_{j}\right)$ in nats per second for the low-SNR regime as

$$
W R_{j} \approx \min \left(\frac{h_{s, r}^{2} P_{s}}{D_{s, r}^{\alpha} N}, \frac{h_{s, j}^{2} P_{s}}{D_{s, j}^{\alpha} N}+\frac{h_{r, j}^{2} P_{r}}{D_{r, j}^{\alpha} N}\right)
$$

where we recall that we consider non-coherent communication, i.e. the correlation coefficient between $X_{s}$ and $X_{r}$ is zero.

We can strengthen this result by observing that if $h_{s, j}^{2} / D_{s, j}^{\alpha}>h_{s, r}^{2} / D_{s, r}^{\alpha}$, then destination $j$ can decode the message without waiting for the relay to transmit. In other words, we have

$W R_{j} \approx \max \left(\frac{h_{s, j}^{2} P_{s}}{D_{s, j}^{\alpha} N}, \min \left(\frac{h_{s, r}^{2} P_{s}}{D_{s, r}^{\alpha} N}, \frac{h_{s, j}^{2} P_{s}}{D_{s, j}^{\alpha} N}+\frac{h_{r, j}^{2} P_{r}}{D_{r, j}^{\alpha} N}\right)\right)$.

We analyze this strategy for $\mathcal{N}=\left\{s, r, t_{1}, t_{2}\right\}$ and compare the rates with the routing scheme.

(i) Schedule with no interference: Fig. 7 shows the schedule where now the destination nodes always listen, which was not the case for routing strategy.

The schedule with interference is similar to the schedule in Fig. 5 with the difference again being that the destination nodes always listen.

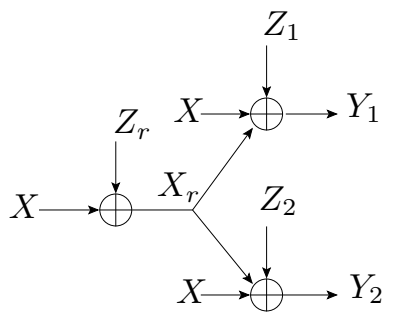

(a)

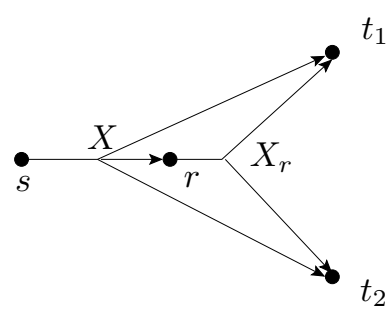

(b)
Fig. 6: Multicast Relay Channel model for $\left\{s, r, t_{1}, t_{2}\right\}$ case.

\begin{tabular}{c||ccc||ccc} 
Time $\backslash$ Node & $s$ & $r$ & Time $\backslash$ Node & $r$ & $t_{1}$ & $t_{2}$ \\
\hline$i$ & 1 & 0 & $i$ & 1 & 1 & 1 \\
$i+1$ & 0 & 1 & $i+1$ & 0 & 1 & 1
\end{tabular}

(a) Transmission.

(b) Reception.

Fig. 7: Schedule using network coding with no interference. The circled positions mark the difference in listening as compared to the routing strategy in Fig. $4 b$.

\section{A. Relay Positioning for Network Coding DF}

Let $F_{\mathcal{N}, N C}$ and $F_{\mathcal{N}, N C}^{*}$ denote, respectively, the multicast flow and its supremum for the network coding scheme. Given $\mathcal{N}$, the flow maximization problem can be written as follows.

$$
\begin{aligned}
& \text { Maximize } F_{\mathcal{N}, N C} \\
& \text { Subject to: }\left\{\frac{h_{s, j}^{2} P_{s}}{D_{\mathcal{N}, N C}^{\alpha} N},\right. \\
&\left.\min \left(\frac{h_{s, r}^{2} P_{s}}{D_{s, r}^{\alpha} N}, \frac{h_{s, j}^{2} P_{s}}{D_{s, j}^{\alpha} N}+\frac{h_{r, j}^{2} P_{r}}{D_{r, j}^{\alpha} N}\right)\right\} .
\end{aligned}
$$

Observe that Program (A) doesn't need a hypergraph as an input. In fact, the relay position $\mathbf{x}_{r}$ is the only optimizing variable, while for routing one must jointly optimize the power and position variables. These differences make the relay positioning problem much easier to solve for the network coding strategy. Next we prove that the function $F_{\mathcal{N}, N C}$ is quasi-concave in node positions and therefore in $\mathbf{x}_{r}$.

Proposition 1: $F_{\mathcal{N}, N C}$ is quasi-concave in $\mathbf{x}_{u}$ for $u=$ $1,2, . ., m, r$, where

$$
\begin{aligned}
F_{\mathcal{N}, N C}= & \min _{1 \leq j \leq m}\left[\operatorname { m a x } \left\{\frac{h_{s, j}^{2} P_{s}}{N\left\|\mathbf{x}_{s}-\mathbf{x}_{j}\right\|^{\alpha}}, \min \left(\frac{h_{s, r}^{2} P_{s}}{N\left\|\mathbf{x}_{s}-\mathbf{x}_{r}\right\|^{\alpha}},\right.\right.\right. \\
& \left.\left.\left.\frac{h_{s, j}^{2} P_{s}}{N\left\|\mathbf{x}_{s}-\mathbf{x}_{j}\right\|^{\alpha}}+\frac{h_{r, j}^{2} P_{r}}{N\left\|\mathbf{x}_{r}-\mathbf{x}_{j}\right\|^{\alpha}}\right)\right\}\right] .
\end{aligned}
$$

We defer the proof of Proposition 1 to Appendix VI-A. Note that a direct consequence of Proposition 1 is that the Program (A) is a quasi-concave optimization problem.

Another interesting consequence of Proposition 1 is presented in Corollary 1. Let the convex hull polygon of the node positions in $\mathcal{N} \backslash\{r\}$ be denoted by $\mathcal{C}$ and $\hat{\mathcal{V}} \subseteq \mathcal{N}$ denote the set of vertices of $\mathcal{C}$. Then let $\mathcal{V}=\hat{\mathcal{V}} \cup\{s, r\}$ and consider solving the problem of relay positioning for $\mathcal{N}$ and 


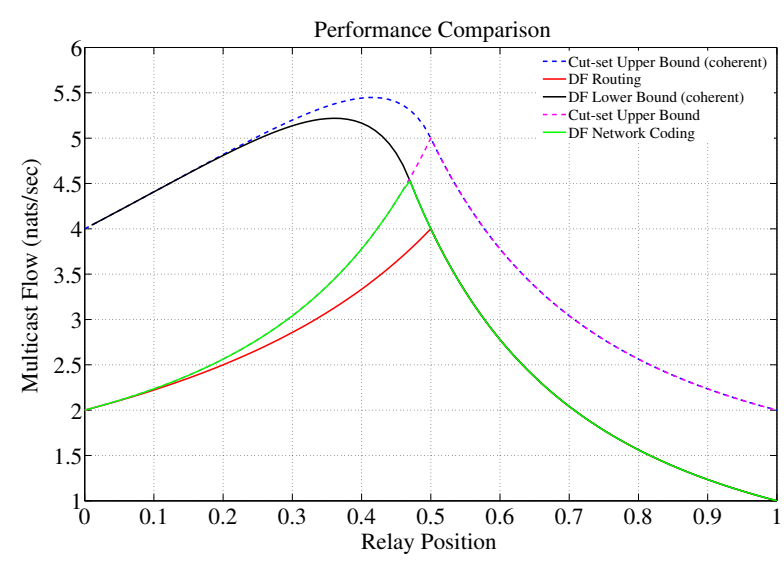

(a)

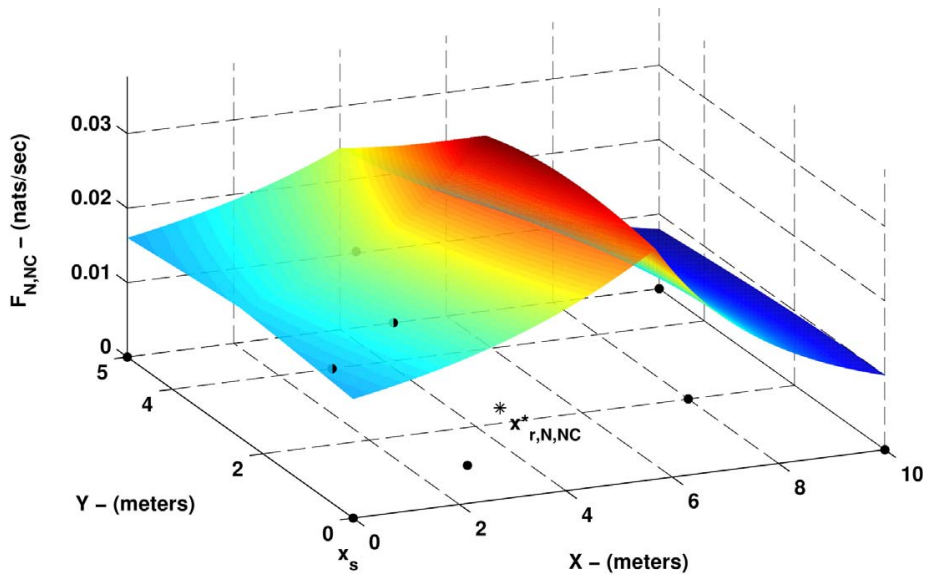

(b)

Fig. 8: (a): Rates for different strategies are plotted for the relay positions in $[0,1]$. (b): $F_{\mathcal{N}, N C}$ for $|\mathcal{N}|=9$. Solid circles represent the nodes and the source is positioned at $(0,0)$. The star marks the optimal relay position $x_{r, \mathcal{N}, N C}^{*}$ achieving $F_{\mathcal{N}, N C}^{*}$.

$\mathcal{V}$ separately. Denote by $\mathbf{x}_{r, \mathcal{N}}^{*}$ and $\mathbf{x}_{r, \mathcal{V}}^{*}$ the relay positions maximizing $F_{\mathcal{N}, N C}$ and $F_{\mathcal{V}, N C}$, respectively. We have the following corollary.

Corollary 1: $F_{\mathcal{N}, N C}^{*}=F_{\mathcal{V}, N C}^{*}$ and $\mathbf{x}_{r, \mathcal{N}}^{*}=\mathbf{x}_{r, \mathcal{V}}^{*}$. The proof is deferred to Appendix VI-B. Corollary 1 implies that to maximize $F_{\mathcal{N}, N C}$ we can ignore all the destination nodes in the set $T$ that are not vertices of $\mathcal{C}$. As a special case, suppose the convex hull of $\mathcal{N} \backslash\{r\}$ is a rectangle and $s$ is positioned at one of its vertices. Then $|\mathcal{V}|=5(\mathcal{V}=$ $\left.\left\{s, r, t_{u}, t_{v}, t_{w}\right\}\right)$ and solving for $F_{\mathcal{V}, N C}^{*}$ will give us $\mathbf{x}_{r, \mathcal{N}}^{*}$ and $F_{\mathcal{N}, N C}^{*}$. Corollary 1 also implies that $\mathbf{x}_{r, \mathcal{N}}^{*}$ and $F_{\mathcal{N}, N C}^{*}$ are insensitive to changes in network topology if the position of the source and the convex hull $\mathcal{C}$ remains unchanged.

\section{Numerical Results}

We compare the achievable rates of the two strategies in the low-SNR regime for a simple set-up of one destination, i.e., $\mathcal{N}=\{s, r, t\}$. The schedule with no-interference is considered. Suppose that the source $s$ and destination $t$ are positioned at 0 and 1 , respectively, and the relay is allowed to be positioned at any point in $[0,1]$ (refer Fig. 8a). The hypergraph for the routing DF strategy has the hyperarc set $\mathcal{A}=\{(s, r),(s, r, t),(r, t)\}$. We use $P_{s}=P_{r}=N=1$, $a_{i, j}=b_{i, j}=c_{i, j}=\frac{1}{D_{i, j}^{\alpha}}$ and the path-loss exponent $\alpha=2$. Figure 8a shows the rates achieved by the two strategies, the routing strategy in red and the network coding strategy in green. The cut-set upper bounds for the general case and the non-coherent case are shown by the dashed blue and dashed magenta curves, respectively. Network coding DF strategy peaks at $F_{\mathcal{N}, N C}^{*} \approx 4.58$ nats $/ \mathrm{sec} / \mathrm{Hz}$, a little before the routing DF strategy which peaks at $F_{\mathcal{N}, R}^{*}=4$ nats $/ \mathrm{sec} / \mathrm{Hz}$. We thus gain approximately $13 \%$ in rate. Also, $x_{r, \mathcal{N}, R}^{*}=0.5$ because solving Program (A) results in sending all the flow through the path $\{(s, r),(r, t)\}$. On the other hand $x_{r, \mathcal{N}, N C}^{*}$ is closer to the source compared to $x_{r, \mathcal{N}, R}^{*}$.

As the relay gets closer to the destination the difference between the two schemes vanishes and the curves merge with the decode-and-forward lower bound. In fact, beyond the middle point (0.5) they achieve the same rates. Intuitively, this can be explained as follows. As the relay gets closer to the destination and farther from the source, the difference of mutual informations between $s$ and $r$ and $s$ and $t$ decreases, resulting in approximately the same signals received at $r$ and $t$ in the first time slot. Thus when the relay transmits the destination does not gain much. Therefore, beyond a certain relay position both the strategies are limited by the relay.

Now consider a plane with 7 destinations $(|\mathcal{N}|=9)$ where the source is positioned at the origin $(0,0)$ and the system parameters are as before. Suppose that the farthest destination from the source is positioned at $(10,5)$. Figure $8 \mathrm{~b}$ plots the multicast flow function $F_{\mathcal{N}, N C}$ and shows that $F_{\mathcal{N}, N C}$ forms a quasi-concave envelope. The relay position $x_{r, \mathcal{N}, N C}^{*}$ maximizing $F_{\mathcal{N}, N C}$ is closer to the source as compared to the farthest destination at $(10,5)$, as expected.

\section{CONCLUSION}

We presented a network coding based DF approach for the Gaussian MRC and compare it with a routing based DF strategy showing a gain of $13 \%$ in the low-SNR regime. For this strategy, we show that the multicast flow function is quasiconcave in the position of relay. As a result all the destination nodes that are not the vertices of the convex hull polygon of the node set $\mathcal{N} \backslash\{r\}$ can be ignored. This has the practical advantage of reducing the size of the optimization problem.

We expect that for low-SNR the rates achieved by the network coding based DF strategy achieve strictly larger rates than amplify-and-forward and compress-and-forward at the optimal relay position. This is the focus of future work.

\section{ACKNOWLEDGEMENTS}

M. Thakur and G. Kramer were supported by the German Ministry of Education and Research in the framework of an Alexander von Humboldt Professorship.

\section{REFERENCES}

[1] V. Aggarwal, A. Bennatan, and A. R. Calderbank, "On Maximizing Coverage in Gaussian Relay Networks," IEEE Trans. Inf. Theory, vol. 55, no. 6, pp. 2518-2536, June 2009. 
[2] G. Kramer, M. Gastpar, and P. Gupta, "Cooperative strategies and capacity theorems for relay networks," IEEE Trans. Inf. Theory, vol. 51, no. 9 , Sept. 2005.

[3] M. Thakur, N. Fawaz, and M. Médard, "Reducibility of joint relay positioning and flow optimization problem," Proc. IEEE Int. Symp. Information Theory, Boston, MA, 2012.

[4] T. M. Cover and A. E. Gamal, "Capacity theorems for the relay channel," IEEE Trans. Inf. Theory, vol. 25, no. 5, pp. 572-584, Sept. 1979.

[5] T. M. Cover, "Broadcast channels," IEEE Trans. Inf. Theory, vol. 18, no. 1, Jan. 1972.

[6] P. P. Bergmans, "A simple converse for broadcast channels with additive white Gaussian noise," IEEE Trans. Inf. Theory, vol. 20, pp. 279-280, Mar. 1974.

[7] S. Boyd and L. Vandenberghe, Convex Optimization. New York: Cambridge University Press, Mar. 2004.

\section{A. Proof of Proposition 1}

\section{APPENDIX}

We use the properties of real valued quasi-concave functions in $\mathbf{R}^{n}$.

Definition 1: A function $f$ in $\mathbf{R}^{n}$ is quasi-concave if for $0 \leq \lambda \leq 1$

$$
f(\theta \mathbf{x}+(1-\theta) \mathbf{y}) \geq \min (f(\mathbf{x}), f(\mathbf{y})), \quad \mathbf{x}, \mathbf{y} \in \mathbf{R}^{n} .
$$

Theorem 1: If $f$ and $f_{i}(i \in\{1, . ., m\})$ are quasi-concave in $\mathbf{R}^{n}$, then so are the following compositions:

(a) affine composition $g \circ f=a f+b$ for reals $a \geq 0$ and $b$.

(b) maximum composition $g \circ f=\max (a, f)$, for real $a \geq 0$.

(c) minimum composition $g=\min _{i \in\{1, . ., m\}} f_{i}$.

For the proof of Theorem 1, we refer the reader to section 3.4 of chapter 3 in [7].

Theorem 2: Let $f$ be quasi-concave in $\mathbf{R}^{n}$ and let $\mathcal{C}$ be the convex hull polyhedron of $m$ points in the set $\mathcal{X}=$ $\left\{\mathbf{x}_{1}, . ., \mathbf{x}_{m}\right\}$ in $\mathbf{R}^{n}$. Then for $\mathbf{x} \in \mathcal{C}$

$$
f(\mathbf{x}) \geq \min _{i \in\{1, . ., m\}} f\left(\mathbf{x}_{i}\right) .
$$

Proof: For any point $\mathbf{x} \in \mathcal{C}$, there is a line segment $\mathbf{x}_{j}$ $\mathbf{y}$ (where $j \in\{1, . ., m\}$ and $\mathbf{y}$ is a point on a face of $\mathcal{C}$ ) containing $\mathbf{x}$, i.e., we have $\mathbf{x}=\theta \mathbf{x}_{j}+(1-\theta) \mathbf{y}$ for some $\theta \in[0,1]$. Using Definition 1 , we get

$$
f(\mathbf{x}) \geq \min \left(f\left(\mathbf{x}_{j}\right), f(\mathbf{y})\right) .
$$

But, $f\left(\mathbf{x}_{j}\right) \geq \min _{i \in\{1, . ., m\}} f\left(\mathbf{x}_{i}\right) \Longrightarrow f(\mathbf{x}) \geq \min _{i \in\{1, . ., m\}} f\left(\mathbf{x}_{i}\right)$, and this completes the proof.

Lemma 1: $\frac{1}{\|\mathbf{x}-\mathbf{y}\|^{\alpha}}$ is quasi-concave in $\mathbf{x}$ and $\mathbf{y}$ for $\alpha \geq 2$.

Proof: Let $\mathbf{z}=\mathbf{x}-\mathbf{y}, f(\mathbf{z})=\|\mathbf{z}\|^{\alpha}$ and $\theta \in[0,1]$. Then from the triangle inequality in $\mathbf{R}^{n}$, for any $\overline{\mathbf{z}}$ and $\hat{\mathbf{z}}$ we have

$$
\begin{aligned}
\|\theta \overline{\mathbf{z}}+(1-\theta) \hat{\mathbf{z}}\| \leq\|\theta \overline{\mathbf{z}}\|+\|(1-\theta) \hat{\mathbf{z}}\| & =\theta\|\overline{\mathbf{z}}\|+(1-\theta)\|\hat{\mathbf{z}}\| \\
& \leq \max (\|\overline{\mathbf{z}}\|,\|\hat{\mathbf{z}}\|) .
\end{aligned}
$$

This implies, $\|\theta \overline{\mathbf{z}}+(1-\theta) \hat{\mathbf{z}}\|^{\alpha} \leq \max \left(\|\overline{\mathbf{z}}\|^{\alpha},\|\hat{\mathbf{z}}\|^{\alpha}\right)$ and

$$
\frac{1}{f(\theta \overline{\mathbf{z}}+(1-\theta) \hat{\mathbf{z}})} \geq \min \left(\frac{1}{f(\overline{\mathbf{z}})}, \frac{1}{f(\hat{\mathbf{z}})}\right) \text {. }
$$

This completes the proof.

Recall that $D_{i, j}=\left\|\mathbf{x}_{i}-\mathbf{x}_{j}\right\|$, for $\mathbf{x}_{j}$ and $\mathbf{x}_{j}$ in $\mathbf{R}^{n}$.
Proof of Proposition 1: For a given node set $\mathcal{N}=$ $\{s, r, T\}$, the flow function $f_{j}$ (for destination $t_{j} \in T$ ) is

$$
f_{j}=\max \left(\frac{h_{s, j}^{2} P_{s}}{N D_{s, j}^{\alpha}}, \min \left(\frac{h_{s, r}^{2} P_{s}}{N D_{s, r}^{\alpha}}, \frac{h_{s, j}^{2} P_{s}}{N D_{s, j}^{\alpha}}+\frac{h_{r, j}^{2} P_{r}}{N D_{r, j}^{\alpha}}\right)\right) .
$$

Then from Lemma 1 and Theorem 1 (a) we know that

$$
\frac{h_{r, j}^{2} P_{r}}{N D_{r, j}^{\alpha}} \text { and } \frac{h_{s, j}^{2} P_{s}}{N D_{s, j}^{\alpha}}+\frac{h_{r, j}^{2} P_{r}}{N D_{r, j}^{\alpha}}
$$

are quasi-concave in $\mathbf{x}_{r}$. Note that $\frac{h_{s, j}^{2} P_{s}}{N D_{s, j}^{\alpha}}$ is a positive real.

Using Theorem 1 (a)-(c), we find that $f_{j}$ is quasi-concave in $\mathbf{x}_{r}$. Finally, using Theorem 1 (c) again, we conclude that

$$
F_{\mathcal{N}, N C}=\min _{1 \leq j \leq m} f_{j}
$$

is quasi-concave in $\mathbf{x}_{r}$. A similar line of argument holds for showing $F_{\mathcal{N}, N C}$ is quasi-concave in $\mathbf{x}_{k}$ for any $k \in \mathcal{N}$.

\section{B. Proof of Corollary 1}

Proof of Corollary 1: Let $\mathcal{C}$ be the convex hull polyhedron of set $\mathcal{N}$ and let $\mathcal{V}$ be the set of its vertices. As $\mathcal{V} \subseteq \mathcal{N}$ we have

$$
F_{\mathcal{N}, N C} \leq F_{\mathcal{V}, N C}
$$

Any point $\mathrm{x} \in \mathcal{C}$ can be expressed as the convex combination of points in $\mathcal{V}=\left\{\mathrm{x}_{1}^{\prime}, . ., \mathrm{x}_{K}^{\prime}\right\}$, i.e., we have

$$
\mathbf{x}=\sum_{k=1}^{K} \lambda_{k} \mathbf{x}_{k}^{\prime}, \quad \lambda_{k} \geq 0, \quad \sum_{k=1}^{K} \lambda_{k}=1 .
$$

Fix the relay position as $\mathbf{x}_{r}$ and consider a destination that is positioned at $\mathbf{x}$, then from Proposition 1 we know that the flow function $f$ is quasi-concave in $\mathbf{x}$. Using Theorem 2, we get

$$
\begin{aligned}
f(\mathbf{x}) & \geq \min _{1 \leq k \leq K} f\left(\mathbf{x}_{k}^{\prime}\right), \\
f(\mathbf{x}) & \geq F_{\mathcal{V}, N C}, \\
F_{\mathcal{N}, N C} & \geq F_{\mathcal{V}, N C} .
\end{aligned}
$$

From inequalities (16) and (19), it follows that

$$
F_{\mathcal{N}, N C}=F_{\mathcal{V}, N C}
$$

Equality (20) holds for any relay position $\mathbf{x}_{r}$ and thus holds for the position maximizing $F_{\mathcal{N}, N C}$, therefore $\mathbf{x}_{r, \mathcal{N}}^{*}=\mathbf{x}_{r, \mathcal{V}}^{*}$. 